\title{
Análise de frações de fibra alimentar em cultivares de feijão cultivadas em dois ambientes
}

\author{
Analysis of dietary fiber fractions in common bean cultivars in different locations
}

\author{
Patrícia Medianeira Grigoletto Londero ${ }^{\mathrm{I}}$ Nerinéia Dalfollo Ribeiro ${ }^{\mathrm{I}^{*}}$ Nerison Luis Poersch $^{\mathrm{I}}$ \\ Irajá Ferreira Antunes ${ }^{\mathrm{II}}$ José Laerte NörnbergIII
}

\begin{abstract}
O objetivo deste trabalho foi determinar as frações de fibra alimentar em cultivares de feijão obtidas em diferentes locais, com vistas à avaliação dos efeitos da interação cultivares $x$ locais. A fibra alimentar foi determinada pelo método enzimático gravimétrico em grãos de 19 cultivares de feijão, obtidos na safra de 2004/2005, em dois municípios do Estado do Rio Grande do Sul. Interações cultivares $x$ locais significativas foram observadas em relação aos teores de fibra alimentar total, à fibra insolúvel, à fibra solúvel e ao rendimento de grãos. A fibra alimentar total variou de 20,85\% ("Macotaço", em Santa Maria) a 31,35\% ("Macanudo", em Pelotas). As cultivares "Macanudo" e "Guateian 6662" apresentam elevados teores de fibra alimentar total, insolúvel e solúvel, e a utilização dessas cultivares como alimento funcional nas dietas deverá ser investigada.
\end{abstract}

Palavras-chave: Phaseolus vulgaris L., interação genótipo $x$ ambiente, alimento funcional.

\section{ABSTRACT}

The objective of this study was to estimate the dietary fiber fractions in common bean cultivars obtained in different locations, for evaluation of the genotype $x$ environment interaction effects. The dietary fiber was determined by enzymatic gravimetric method in grains of the nineteen common bean cultivars, obtained in 2004/2005 crop season, in two locations of Rio Grande do Sul State, Brazil. Significant genotype $x$ environment interactions were detected for total, insoluble and soluble dietary fiber content and grain yield. The total dietary fiber content varied from 20.85\% ('Macotaço', in Santa Maria) to $31.35 \%$ ('Macanudo', in Pelotas). The 'Macanudo' and 'Guateian 6662' cultivars showed high total, insoluble and soluble dietary fiber content and its utilization as a regular food in diets can be studied.

\author{
Key words: Phaseolus vulgaris L., genotype $x$ environment \\ interaction, regular food.
}

O feijão (Phaseolus vulgaris L.) apresenta teores significativos de proteínas, carboidratos, vitaminas, minerais e fibra e apresenta baixo conteúdo de gordura e de colesterol (HOSFIELD, 1991; MORROW, 1991), por isso ele é considerado de alta qualidade nutricional e funcional.

Além disso, o feijão é o alimento de origem vegetal que apresenta maior teor de fibra alimentar quando comparado aos cereais (trigo, arroz e milho) e a várias hortaliças (ACEVEDO \& BRESSANI, 1990). Valores de 33,39 a 39,39\% de fibra alimentar foram observados em cultivares e em linhagens segregantes de feijão desenvolvidas no Brasil (LONDERO et al., 2005; LONDERO et al., 2006), superando os percentuais de 7,45 a 10,25\% verificados em 102 genótipos de aveia (SILVA et al., 2006). A utilização de alimentos ricos em fibra na dieta é recomendável para a regulação do funcionamento do trato gastrointestinal e para o controle e/ou prevenção de certas doenças crônicas e degenerativas (RAUPP et al., 1999), devido aos efeitos metabólicos e fisiológicos proporcionados ao organismo.

A fibra alimentar é composta pela fração solúvel - substâncias pécticas, gomas, algumas hemiceluloses e ß-glucanas, e pela fração insolúvel celulose, lignina e a maioria das hemiceluloses (Guerra

IDepartamento de Fitotecnia, Universidade Federal de Santa Maria (UFSM), Campus Universitário, 97105-900, Santa Maria, RS, Brasil. E-mail: neiadr@smail.ufsm.br. *Autor para correspondência.

${ }^{\text {IE} E m b r a p a ~ C l i m a ~ T e m p e r a d o, ~ P e l o t a s, ~ R S, ~ B r a s i l . ~}$

IIIDepartamento de Ciência e Tecnologia dos Alimentos, UFSM, Santa Maria, RS, Brasil. 
et al., 2004). A fração solúvel atua como importante agente hipoglicemiante e hipocolesterolemiante, ajudando no controle dos níveis de glicose no sangue em diabéticos e na prevenção de doenças cardiovasculares; a fração insolúvel auxilia na prevenção de câncer de cólon e de problemas intestinais (TOPPING, 1991; MOORE et al., 1998). O feijão é um dos poucos alimentos integrais que possui conteúdo balanceado de ambas as frações da fibra (HUGHES, 1991), por isso seu consumo como alimento funcional deve ser melhor investigado.

O conteúdo de fibra alimentar e de suas frações é variável geneticamente e pode ser explorado pelo melhoramento. Assim, LONDERO et al. (2006) obtiveram variação de 24,82 a 31,35\% para fibra insolúvel (FI) e de 8,04 a 11,11\% para a fibra solúvel (FS), ou seja, aproximadamente 3/4 da fibra alimentar em grãos de feijão preto é fração insolúvel.

Para que seja possível o desenvolvimento de germoplasma que possa ser utilizado com finalidade específica na composição das dietas, se faz necessário o conhecimento dos efeitos da interação genótipo $\mathrm{x}$ ambiente sobre o teor de fibra alimentar e de suas frações em cultivares de feijão. Estas qualidades nutricionais e tecnológicas do feijão são determinadas em parte pelo genótipo e influenciadas pelas condições do ambiente durante o desenvolvimento da planta e dos grãos (DALLA CORTE et al., 2003; LEMOS et al., 2004). Por isso, o objetivo desse trabalho foi avaliar o efeito da interação cultivares $\mathrm{x}$ locais sobre os teores de fibra alimentar total, insolúvel e solúvel, e o rendimento de grãos de cultivares de feijão disponíveis para o cultivo no Brasil.

O experimento foi conduzido no Núcleo Integrado de Desenvolvimento em Análises Laboratoriais (NIDAL), da Universidade Federal de Santa Maria (UFSM), Santa Maria - RS. Os grãos utilizados foram obtidos na safra agrícola de 2004/2005, em áreas experimentais dos Programas de Melhoramento da UFSM e da Embrapa Clima Temperado, Pelotas - RS.

Em Santa Maria, o experimento foi realizado no Departamento de Fitotecnia, da UFSM, na região da depressão central do RS, a 95m de altitude, latitude $29^{\circ} 42^{\prime}$ S e longitude $53^{\circ} 43^{\prime} \mathrm{W}$. O clima da região é do tipo Cfa, temperado chuvoso, com chuvas bem distribuídas ao longo dos anos, e subtropical do ponto de vista térmico, e o solo é classificado como Argissolo Bruno-Acinzentado alítico típico. Em Pelotas, por sua vez, o experimento foi conduzido em área da Embrapa Clima Temperado, na região sul do Estado do RS, a $17 \mathrm{~m}$ de altitude, latitude $31^{\circ} 7^{\prime} \mathrm{S}$ e longitude $52^{\circ} 1^{\prime} \mathrm{W}$. O clima também é subtropical do tipo Cfa e o solo da região é caracterizado como Cambissolo Háplico distrófico típico.

No campo, o solo foi preparado de maneira convencional e a adubação foi realizada de acordo com a interpretação da análise química do solo. O controle de insetos foi realizado com a aplicação de Metamidofós e o controle de plantas invasoras foi manual e efetuado sempre que necessário, de maneira que a cultura não sofresse competição.

O delineamento experimental de blocos ao acaso, com três repetições, foi utilizado no campo em ambos os locais e as 19 cultivares de feijão foram avaliadas (Tabela 1). As parcelas foram compostas de quatro fileiras de $4 \mathrm{~m}$ de comprimento, espaçadas de $0,50 \mathrm{~m}$, e a área útil de 3,0 $\mathrm{m}^{2}$. A colheita e a trilha das plantas foram realizadas manualmente na maturação fisiológica e, após a retirada das impurezas, os grãos foram secados ao sol e em estufa $\left(65\right.$ a $\left.70^{\circ} \mathrm{C}\right)$, até umidade média de $13 \%$, quando foi determinado o rendimento de grãos. Em seguida, amostras de $100 \mathrm{~g}$ de grãos foram moídas em micromoinho até a obtenção de partículas inferiores a $1 \mathrm{~mm}$. As análises de fibra alimentar foram realizadas em duas repetições, em duplicata, de acordo com a metodologia descrita pela AOAC INTERNACIONAL (1995).

Os dados obtidos foram submetidos à análise da variância, utilizando o teste $\mathrm{F}$ a $5 \%$, para testar as hipóteses dos efeitos principais e das interações. O efeito de cultivares foi considerado como fixo e o efeito de locais foi considerado aleatório. A comparação das médias foi realizada pelo teste de ScottKnott, a 5\% de probabilidade de erro, para as variáveis com interação cultivares x locais. As análises estatísticas foram efetuadas de acordo com o modelo de blocos ao acaso, para o rendimento de grãos, e inteiramente casualizado, para a fibra alimentar.

$\mathrm{Na}$ análise de variância, foram obtidas interações cultivares x locais significativas em relação aos teores de fibra alimentar total, insolúvel e solúvel, e ao rendimento de grãos, evidenciando resposta diferenciada das cultivares de feijão aos locais de avaliação. Entretanto, o teor de fibra crua de acessos de feijão avaliados em três safras agrícolas na Espanha, não foi afetado pela interação genótipos x ambientes (ESCRIBANO et al., 1997). As diferenças resultantes do tipo de solo e das condições climáticas entre esses países, provavelmente, tenham contribuído para as variações observadas nos teores de fibra.

Os teores de fibra alimentar total variaram de 22,05\% (“Iapar 31”) a 31,35\% (“Macanudo”) em Pelotas (Tabela 1). Entretanto, em Santa Maria foram observados valores entre 20,85\% ("Macotaço") e 26,22\% ("Guateian 6662"). Esses valores foram inferiores aos obtidos anteriormente em cultivares e 
Tabela 1 - Médias dos teores de fibra alimentar total, insolúvel e solúvel, e rendimento de grãos de 19 cultivares de feijão avaliadas em dois locais (Pelotas e Santa Maria), no Estado do Rio Grande do Sul.

\begin{tabular}{|c|c|c|c|c|c|c|c|c|c|c|c|c|}
\hline \multirow{3}{*}{ Cultivar } & \multicolumn{3}{|c|}{ Fibra total $(\%)\left({ }^{1}\right)$} & \multicolumn{3}{|c|}{ Fibra insolúvel (\%) } & \multicolumn{3}{|c|}{ Fibra solúvel (\%) } & \multicolumn{3}{|c|}{ Rendimento (kg ha ${ }^{-1}$ ) } \\
\hline & & Santa & & & Santa & & & Santa & & & Santa & \\
\hline & Pelotas & Maria & Média & Pelotas & Maria & Média & Pelotas & Maria & Média & Pelotas & Maria & Média \\
\hline “Macanudo” & 31,35 a & 25,38 a & 28,36 & 26,89 a & $21,16 \mathrm{~b}$ & 24,02 & $4,46 \mathrm{~b}$ & 4,22 a & 4,34 & $2160 \mathrm{~b}$ & $2163 b$ & 2161 \\
\hline “Minuano” & $29,00 \mathrm{~b}$ & $24,88 \mathrm{~b}$ & 26,94 & $25,88 \mathrm{~b}$ & $21,58 \mathrm{~b}$ & 23,73 & $3,12 \mathrm{c}$ & $3,31 \mathrm{~b}$ & 3,21 & $2030 \mathrm{~b}$ & $2013 \mathrm{~b}$ & 2021 \\
\hline "Pérola” & 28,92 b & $22,93 \mathrm{c}$ & 25,93 & $25,56 \mathrm{~b}$ & $21,33 \mathrm{~b}$ & 23,44 & $3,36 \mathrm{c}$ & $1,61 \mathrm{~b}$ & 2,48 & 2304 a & 1710 c & 2007 \\
\hline “Macotaço” & $27,91 \mathrm{c}$ & $20,85 \mathrm{~d}$ & 24,38 & $24,91 \mathrm{~b}$ & $19,15 \mathrm{~d}$ & 22,03 & $3,00 \mathrm{c}$ & $1,70 \mathrm{~b}$ & 2,35 & 2037 b & 1685 c & 1861 \\
\hline “Guateian 6662” & $27,10 \mathrm{c}$ & 26,22 a & 26,66 & $19,99 \mathrm{~g}$ & 23,51 a & 21,75 & 7,11 a & $2,71 \mathrm{~b}$ & 4,91 & 2148 b & 1588 c & 1868 \\
\hline “G. Brilhante” & 26,98 c & $24,24 \mathrm{~b}$ & 25,61 & $24,07 \mathrm{c}$ & $19,15 \mathrm{~d}$ & 21,61 & $2,90 \mathrm{c}$ & 5,09 a & 4,00 & $2090 \mathrm{~b}$ & $2305 \mathrm{~b}$ & 2197 \\
\hline “TPS Nobre” & 26,53 c & $24,41 \mathrm{~b}$ & 25,47 & $23,50 \mathrm{c}$ & 20,05 c & 21,78 & $3,03 \mathrm{c}$ & 4,36 a & 3,69 & 2329 a & 2212 b & 2270 \\
\hline “BRS Valente” & $26,48 \mathrm{c}$ & $23,78 \mathrm{~b}$ & 25,13 & $22,76 \mathrm{~d}$ & $20,56 \mathrm{c}$ & 21,66 & $3,73 \mathrm{c}$ & $3,22 \mathrm{~b}$ & 3,48 & 2098 b & 1994 b & 2046 \\
\hline "Rio Tibagi” & 26,32 c & 23,77 b & 25,04 & $24,70 \mathrm{~b}$ & 19,93 с & 22,32 & $1,61 \mathrm{c}$ & $3,84 \mathrm{a}$ & 2,72 & $2163 \mathrm{~b}$ & 1572 c & 1868 \\
\hline “D. Negro” & $26,14 \mathrm{c}$ & $24,29 \mathrm{~b}$ & 25,22 & $22,64 \mathrm{~d}$ & $20,73 \mathrm{c}$ & 21,68 & $3,50 \mathrm{c}$ & $3,57 \mathrm{~b}$ & 3,53 & $2038 \mathrm{~b}$ & $1710 \mathrm{c}$ & 1874 \\
\hline “BRS Expedito” & $25,77 \mathrm{~d}$ & $24,82 \mathrm{~b}$ & 25,29 & $20,37 \mathrm{f}$ & 20,29 c & 20,33 & $5,40 \mathrm{~b}$ & 4,53 a & 4,96 & 2354 a & 2014 b & 2184 \\
\hline “Carioca” & $25,10 \mathrm{~d}$ & $22,93 \mathrm{c}$ & 24,02 & $21,71 \mathrm{e}$ & 20,32 c & 21,01 & $3,39 \mathrm{c}$ & $2,61 \mathrm{~b}$ & 3,00 & 2317 a & 1844 c & 2080 \\
\hline “TPS Bionobre” & $25,03 \mathrm{~d}$ & $23,96 \mathrm{~b}$ & 24,49 & $19,95 \mathrm{~g}$ & $21,33 \mathrm{~b}$ & 20,64 & $5,08 \mathrm{~b}$ & $2,63 \mathrm{~b}$ & 3,86 & 2430 a & $2136 \mathrm{~b}$ & 2283 \\
\hline “Iraí” & $24,91 \mathrm{~d}$ & $24,49 \mathrm{~b}$ & 24,70 & $20,39 \mathrm{f}$ & 20,00 c & 20,19 & $4,52 \mathrm{~b}$ & 4,49 a & 4,50 & 2304 a & $2644 \mathrm{a}$ & 2474 \\
\hline “FTS Soberano” & $24,49 \mathrm{~d}$ & 22,38 c & 23,44 & $19,46 \mathrm{~g}$ & $19,18 \mathrm{~d}$ & 19,32 & $5,03 \mathrm{~b}$ & $3,20 \mathrm{~b}$ & 4,11 & $1920 \mathrm{~b}$ & $2185 \mathrm{~b}$ & 2052 \\
\hline “TPS Bonito” & $24,26 \mathrm{~d}$ & $24,68 \mathrm{~b}$ & 24,47 & $20,83 \mathrm{f}$ & $21,60 \mathrm{~b}$ & 21,22 & $3,43 \mathrm{c}$ & $3,08 \mathrm{~b}$ & 3,25 & 1822 b & 2511 a & 2166 \\
\hline “Iapar 44” & 23,59 e & $22,41 \mathrm{c}$ & 23,00 & $20,60 \mathrm{f}$ & $19,69 \mathrm{~d}$ & 20,15 & $2,99 \mathrm{c}$ & $2,72 \mathrm{~b}$ & 2,85 & $2023 \mathrm{~b}$ & $1163 \mathrm{~d}$ & 1593 \\
\hline "FTS Magnífico" & 23,02 e & 25,55 a & 24,28 & $19,67 \mathrm{~g}$ & $22,06 \mathrm{~b}$ & 20,86 & $3,35 \mathrm{c}$ & $3,49 \mathrm{~b}$ & 3,42 & $1869 \mathrm{~b}$ & 1999 b & 1934 \\
\hline “Iapar 31” & $22,05 \mathrm{f}$ & $24,54 \mathrm{~b}$ & 23,29 & $18,36 \mathrm{~h}$ & $21,49 \mathrm{~b}$ & 19,92 & $3,69 \mathrm{c}$ & $3,05 \mathrm{~b}$ & 3,37 & 2468 a & 2004 b & 2236 \\
\hline Média & 26,05 & 24,03 & 25,04 & 22,22 & 20,69 & 21,46 & 3,83 & 3,34 & 3,58 & 2153 & 1971 & 2062 \\
\hline CV (\%) & 2,53 & 2,39 & 2,43 & 2,33 & 2,48 & 2,58 & 22,15 & 25,64 & 23,8 & 9,37 & 9,69 & 9,53 \\
\hline
\end{tabular}

( $\left.{ }^{1}\right)$ Médias não seguidas por mesmas letras na vertical diferem entre si, a 5\% de probabilidade, pelo teste de Scott-Knott.

em populações segregantes de feijão preto avaliadas em Santa Maria (LONDERO et al., 2005; LONDERO et al., 2006).

A fibra insolúvel variou de 18,36\% ("Iapar 31”) a 26,89\% (“Macanudo”), em Pelotas, e de 19,15\% ("Guapo Brilhante” e "Macotaço”) a 23,51\% ("Guateian 6662”), em Santa Maria. As cultivares com maior teor de fibra insolúvel - "Macanudo" e "Guateian 6662”poderiam ser utilizadas em dietas menos calóricas e para a prevenção de constipação, pois essa fração da fibra tem a função de acelerar o tempo de trânsito intestinal, auxiliando na prevenção de doenças do trato gastrointestinal (MOORE et al., 1998; VANDERHOOF, 1998).

Já para a fibra solúvel, amplitude de 1,61\% (“Rio Tibagi”) a 7,11\% (“Guateian 6662”) foi obtida em Pelotas e de 1,61\% ("Pérola”) a 5,09\% ("Guapo Brilhante"), em Santa Maria. As cultivares "Guateian 6662” e "Guapo Brilhante” poderiam ser administradas para o controle do colesterol e para a alimentação de diabéticos devido aos efeitos hipoglicemiante e hipocolesterolemiante da fibra solúvel, ajudando no controle dos níveis de glicose no sangue em diabéticos e na prevenção de doenças cardiovasculares (TOPPING, 1991; MOORE et al., 1998).

Com relação ao rendimento de grãos, a aplicação do teste de Scott-Knott possibilitou a formação de dois grupos em Pelotas (Tabela 1). As cultivares "TPS Nobre”, "BRS Expedito”, “TPS Bionobre”, "Pérola”, “Carioca”, "Iraí” e "Iapar 31” apresentaram elevado rendimento de grãos. Já em Santa Maria, apenas “Iraí” e “TPS Bonito” se destacaram.

A possibilidade de desenvolvimento de cultivares de feijão com elevado potencial de rendimento de grãos e de melhor qualidade nutricional é objetivo do melhoramento. Nesse sentido, a cultivar "Iraí" é promissora para uso na agricultura e na alimentação, pois apresentou superioridade para rendimento de grãos (2644kg ha-1) e para fibra solúvel (4,49\%) em Santa Maria (Tabela 1). 
Assim, considerando que o consumo de feijão pode proporcionar efeitos preventivos e terapêuticos para doenças do coração, diabetes, obesidade e câncer (GEIL \& ANDERSON, 1994), há necessidade de que pesquisas mais aprofundadas comprovem ou não os benefícios para a saúde do consumo de determinada cultivar de feijão. A partir do conhecimento das frações da fibra nos diferentes genótipos, estes podem ser usados de forma direcionada para finalidades específicas na alimentação humana. O grande desafio para o melhoramento genético está na identificação de cultivares de feijão com elevados teores de fibra alimentar total, insolúvel e solúvel, independente do ambiente de cultivo. Assim, constatou-se que a "Macanudo" foi a de maior teor de fibra insolúvel, em Pelotas, e destacou-se em fibra total nos dois locais de avaliação (Tabela 1). "Guateian 6662” foi superior em fibra solúvel, em Pelotas, e foi a de maior fibra insolúvel e enquadrou-se no grupo de maior fibra total, em Santa Maria. As cultivares "Macanudo” e “Guateian 6662” apresentaram superioridade para a fibra alimentar e suas frações nos ambientes avaliados, por isso a possibilidade de uso como alimento funcional deverá ser investigada.

A maior disponibilidade de informações sobre a genética e os efeitos da interação genótipos $\mathrm{x}$ ambientes certamente resultará em avanços no melhoramento da qualidade nutricional dessa leguminosa. Considerando que o feijão é utilizado rotineiramente na composição dos cardápios de parcela significativa da população brasileira, sobretudo nas classes socioeconômicas menos favorecidas, os benefícios proporcionados serão significativos.

\section{AGRADECIMENTOS}

Ao Conselho Nacional de Desenvolvimento Científico e Tecnológico (CNPq), à Coordenação de Aperfeiçoamento de Pessoal de Nível Superior (CAPES) e à Fundação de Amparo a Pesquisa do Estado do Rio Grande do Sul (FAPERGS), pelo auxílio financeiro e pelas bolsas concedidas.

\section{REFERÊNCIAS}

ACEVEDO, E.; BRESSANI, R. Contenido de fibra dietetica y digestibilidad del nitrogeno en alimentos centroamericanos: Guatemala. Archivos Latinoamericanos de Nutricion, Caracas, v.40, n.3, p.439-451, 1990.

AOAC INTERNATIONAL (Gaithersburg, Estados Unidos). Official methods of analysis of AOAC International. 16.ed. Washington, 1995. 2000p.
DALLA CORTE, A. et al. Environment effect on grain quality in early common bean cultivars and lines. Crop Breeding and Applied Biotechnology, Londrina, v.3, n.3, p.193-202, 2003.

ESCRIBANO, M.R. et al. Genetic diversity in pod and seed quality traits of common bean populations from northwestern Spain. Euphytica, Netherlands, v.93, n.1, p.71-81, 1997.

GEIL, P.B.; ANDERSON, J.W. Nutrition and health implications of dry beans: a review. Journal of the American College of Nutrition, Clearwater, v.13, n.6, p.549-558, 1994.

GUERRA, N.B. et al. Modificações do método gravimétrico não enzimático para determinar fibra alimentar solúvel e insolúvel em frutos. Revista de Nutrição, Campinas, v.17, n.1, p.45-52, 2004.

HOSFIELD, G.L. Genetic control of production and food quality factors in dry bean. Food Technology, Chicago, v.45, n.9, p.98-103, 1991 .

HUGHES, J.S. Potential contribution of dry bean dietary fiber to health. Food Technology, Chicago, v.45, n.9, p.122-126, 1991.

LEMOS, L.B. et al. Características agronômicas e tecnológicas de genótipos de feijão do grupo comercial Carioca. Pesquisa Agropecuária Brasileira, Brasília, v.39, n.4, p.319-326, 2004.

LONDERO, P.M.G. et al. Genetic variability for dietary fiber content in common bean populations. Crop Breeding and Applied Biotechnology, Londrina, v.5, n.1, p.86-90, 2005.

LONDERO, P.M.G. et al. Herança dos teores de fibra alimentar e rendimento de grãos em populações de feijoeiro. Pesquisa Agropecuária Brasileira, Brasília, v.41, n.1, p.51-58, 2006.

MOORE, M.A. et al. Soluble and insoluble fiber influences on cancer development. Critical Reviews in Oncology/ Hematology, New York, v.27, n.3, p.229-242, 1998.

MORROW, B. The rebirth of legumes. Food Technology, Chicago, v.45, n.9, p.96-121, 1991.

RAUPP, D.S. et al. Composição e propriedades fisiológico nutritivas de uma farinha rica em fibra insolúvel obtida do resíduo fibroso de fecularia de mandioca. Ciência e Tecnologia de Alimentos, Campinas, v.19, n.2, p.205-210, 1999.

SILVA, C.F.L. et al. Frações de fibra em aveia e sua aplicação em programas de melhoramento. Pesquisa Agropecuária Brasileira, Brasília, v.41, n.6, p.975-980, 2006.

TOPPING, D.L. Soluble fiber polysaccharides: Effects on plasma cholesterol and colonic fermentation. Nutrition Reviews, Catalunya, v.49, n.7, p.195-203, 1991.

VANDERHOOF, J.A. Immunonutrition: the role of carbohydrates. Nutrition, v.14, n.7/8, p.595-598, 1998. 